\title{
Signal-to-Interference Ratio between Atto Cells in an Ultra-High Density Wireless Access Network
}

\author{
G. Vermeeren* \\ G. Torfs ${ }^{\dagger}$ \\ A. Thielens* \\ H. Rogier ${ }^{\dagger}$ \\ P. Demeester ${ }^{\dagger}$ \\ L. Martens* \\ W. Joseph ${ }^{\dagger}$
}

\begin{abstract}
An approach to deal with the demand for increasing bandwidth and data rates from the network is to deploy an ultra-high density access network consisting of a large number of very small cells. In such a wireless network, reducing interference will contribute to meeting the targeted very-high bitrates and low latency. In this study, we evaluated the signal-to-interference ratio in an ultra-high density network consisting of atto cells operating at $3.5 \mathrm{GHz}$ and integrated in floor tiles of $15 \mathrm{~cm}$ by $15 \mathrm{~cm}$ and a moving antenna attached to a robot. We observed that interference in an atto cell can be significantly reduced by altering the polarization of neighboring floor antennas. Moreover, simultaneous communication in neighboring atto cells is possible and depends on the height of the moving antenna and the mutual orientation of the neighboring cells.
\end{abstract}

\section{INTRODUCTION}

A potential approach to deal with the demand for increasing bandwidth and data rates from the network is to deploy an ultra-high density access network consisting of a large number of very small cells each covering only a few $\mathrm{dm}^{2}$, so called atto cells, which can be easily integrated in a floor. This novel wireless architecture (ATTO) was presented in [1]. It aims at providing bitrates of 100 Gbps with latencies below $10 \mu \mathrm{s}$. Simultaneously, communication in the $3.5 \mathrm{GHz}$ will be possible as legacy-mode. A future application of such a network is a distributed robotic system in a factory-of-the-future environment. The small cells allow the necessary bitrates and latencies required by the distributed robots to communicate and (jointly) perform the most difficult tasks. As opposed to future $5 \mathrm{G}$ networks, there will only be one short-range communication link between each moving robot and the single cell just below the robot. One of the requirements to obtain the targeted high bitrate of 100 Gbps is minimizing interference between the small cells. To validate the ATTO concept, and show the potential of noninterfering small cells, we measured interference at

\footnotetext{
${ }^{*}$ Ghent University imec, WAVES, Department of Information Technology, Tech Lane Ghent Science Park Campus A, Technologiepark-Zwijnaarde 15, 9052 Ghent, Belgium, email: gunter.vermeeren@ugent.be, tel.: +3293314895

${ }^{\dagger}$ Ghent University imec, IDLab, Department of Information Technology, Tech Lane Ghent Science Park Campus A, Technologiepark-Zwijnaarde 15, 9052 Ghent, Belgium, email: guy.torfs@ugent.be
}

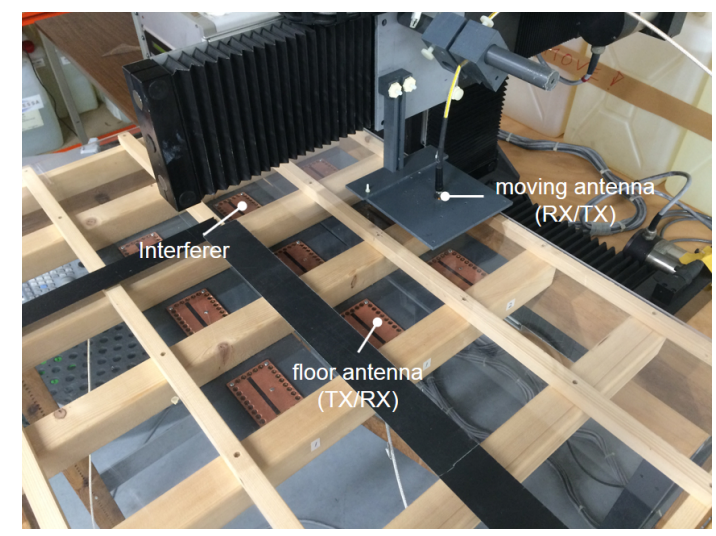

Figure 1: 3x3 atto cell embedded in a floor made of a wooden support and covered by plexiglass. The moving antenna is attached to the automatic positioning system or robot; the interferer is at a distance of two cells from the communicating atto cell.

$3.5 \mathrm{GHz}$. In a previous study [2], we already investigated the exposure from single ATTO cell in terms of electric field and specific absorption rate (SAR).

In the Section 2, we describe the measurement setup, configurations and methodology. Section 3 discusses the results of the interference measurements for the selected configurations and evaluates the orientation of, and the distance between the floor cells. We conclude the paper with a summary of the major outcomes of the study.

\section{MATERIALS AND METHODS}

In the presented study, we experimentaly investigated the interference in atto cells. The atto cells (Figure 1) were embedded in a floor made of a wooden support covered by plexiglass. The floor consisted of $3 \times 3$ atto cells. Each cell contained a linearly polarized planar, substrate-integratedwaveguide (SIW) cavity-backed slot antenna [3], positioned $65 \mathrm{~mm}$ below the top surface of the floor, and radiating upwards. Above the floor, the same type of antenna, but now facing downwards was attached to the automatic positioning system Pythron IXE -C-T (DB, Waregem, Belgium).

Using this setup, we investigated the signal-to- 




(a)

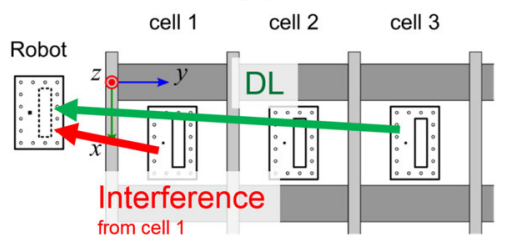

(b)

Figure 2: SIR in (a) uplink and (b) in downlink. The green arrows show the wanted downlink and uplink signal; the red arrows show the interfering signal

interference ratio (SIR) in an atto cell caused by a communicating neighboring cell. The SIR is defined as follows:

$$
S I R=\frac{S_{21, \text { signal }}}{S_{21, \text { interference }}}
$$

with $\mathrm{S}_{21 \text {,signal }}$ and $\mathrm{S}_{21}$,interference the transmission coefficient in the signalling path and interfering path, respectively. We measured $\left(S_{21}\right)$ between communicating and interfering cells using the vector network analyzer ZNB 20, $100 \mathrm{kHz} 20$ GHz (Rohde \& Schwarz, Münich, Germany). Noncommunicating antennas antennas were terminated by a $50 \Omega$ load to avoid additional reflections. We distinguished between SIR in downlink (i.e., at the receiving moving or robot cell) and uplink (i.e., at the receiving floor cell) as shown in Figure 2.

We evaluated the SIR as a function of the horizontal and vertical position of the moving antenna or robot antenna. We investigated the influence of the orientation of neighboring cells and the distance between interferer and communicating cells. Since the antennas were linearly polarized, we evaluated different orientations for neighboring antennas in the floor cells. The examined configuration is denoted by three consecutive letters indicating the orientation of the antenna in the following order: robot - communicating floor cell - interfering floor cell. The antenna is vertically ortiented when the slot of the antenna is paralel to the $\mathrm{x}$-axis, and horizontally when the slot is paralel to the y-axis. As an example, the configuration $\mathrm{VHH}$ is shown in Figure 3.
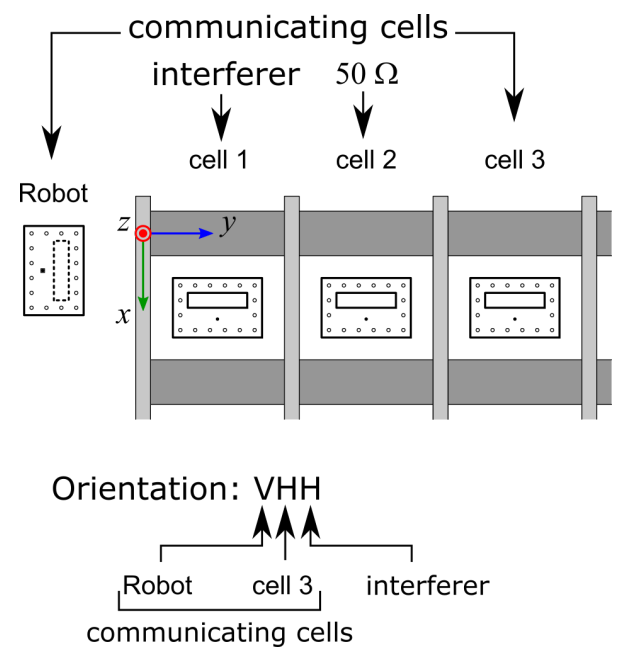

Figure 3: Configuration with interferer at cell 1 and orientation VHH. The letters indicate the orientation of the antenna for consecutively robot, communicating floor cell, and interfering floor cell.

\section{RESULTS}

\subsection{Signal-to-interference ratio}

Figure 4 shows the measured SIR distribution for the configuration VVV (i.e., robot, communicating floor cell, and interfering floor cell are all oriented parallel to the $\mathrm{y}$-axis). The coordinates (x,y,z) represent the position of the robot antenna above the cover of the atto floor. Remark that the scanned area only covered half of the last cell, which is the communicating atto cell, because of the limited reach of our robot system: the robot system can only cover a cube with a side of $400 \mathrm{~mm}$.

The evaluation of the interference in the atto cell is based on the median or 50th percentile $\left(\mathrm{p}_{50}\right)$ of the SIR in a horizontal plane (constant z-value) over the footprint of a single cell. As an example, Figure 5 shows the median SIR for the configuration of Figure 4. The interfering antenna is cell 1 , the communicating antenna is cell 3 , and cell 2 is not radiating (i.e., terminated with a $50 \Omega$ ) load. The SIR is the smallest, largest when the robot antenna is above the interfering cell 1 , communicating cell 3, respectively. With increasing height, SIR drops above communicating cell 3 and increases above interfering cell 1.

\subsubsection{Influence of antenna orientation}

Figure 6 shows the influence of the orientation of the antenna on the SIR in both downlink and uplink signal and for an interer at a distance of one and two cells. The orientation of the interfering cell (cell 2 or 3 ) is changed from vertical to hori- 


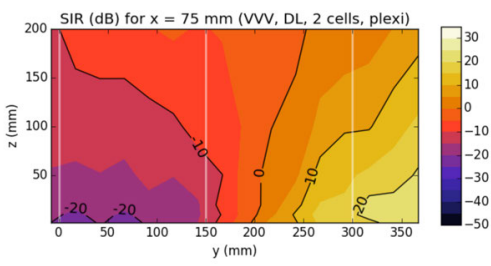

(a)

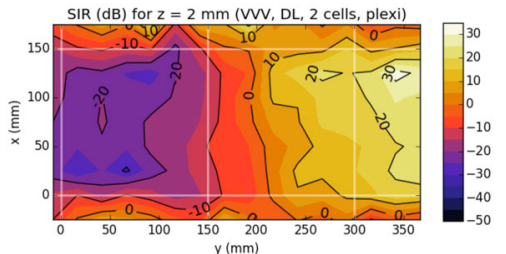

(b)

Figure 4: Downlink SIR distribution in (a) vertical plane in the middle of the atto cell, and (b) horizontal plane $2 \mathrm{~mm}$ above the atto floor plexiglass cover when the robot moves over the floor.



Figure 5: The median of the SIR in a horizontal plane plane above the floor cell. Remark that each cell extends over an area of $(150 \mathrm{~mm} \times 150 \mathrm{~mm})$. The legend indicates the location of the robot, or moving antenna, with respect to the floor cells.

zontal. We observe that if the interferer is oriented perpendicular to the communicating antennas, the SIR increases in both uplink and downlink (by up to $30 \mathrm{~dB}$ ). This is a consequency of the linear polarization of the selected antennas. The difference in polarization between communicating antennas and interfer results in an increase in SIR.

\subsubsection{Influence of antenna distance to in- terferer}

Figure 7 shows the influence of the distance of the interferer on the SIR in both downlink and uplink, for vertical and horizontal orientation of the interferer. When communicating and interfering cells have the same orientation (here: vertical), then we observe that for the uplink the SIR increases with increasing distance between interferer and commu-

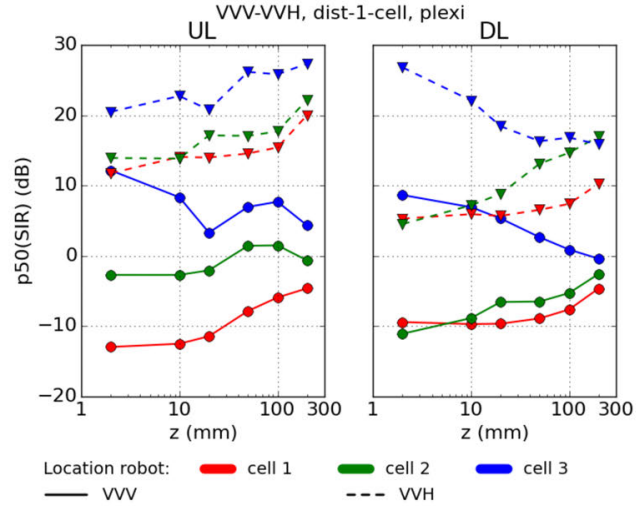

(a)

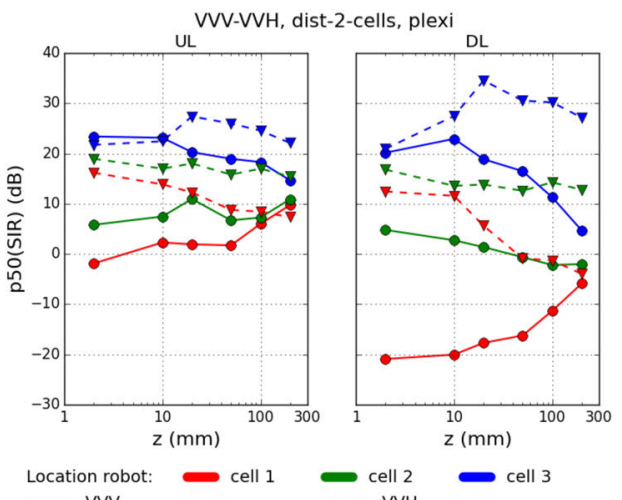

(b)

Figure 6: Influence of the antenna orientation on the median of the SIR over the extent of a single cell in a horizontal plane. The color represents the location of the robot with respect to the floor cells; the line style indicates the orientation of the antenna in the cells as explained in Figure 3.

nicating floor cell. For the downlink, however, we observe a strong interference when the robot is above the interfering cell. When communicating and interfering cells are oriented perpendicularly, then we observe a larger SIR then when the antennas are oriented in the same way due to the cross polarization between interferer and communicating cells. We also observe in Figure7, that the SIR drops significantly ( $\mathrm{p}_{50}(\mathrm{SIR})$ ranging from $-20 \mathrm{~dB}$ to $-6 \mathrm{~dB}$ ) when the interferer is in between the robot and the communicating cell and has the same orientation as the communicating cells.

\section{CONCLUSIONS}

We evaluated the signal-to-interference ratio in an atto cell, an ultra-high density wireless access network with cells integrated in floor tiles. The investigated atto cells operated at $3.5 \mathrm{GHz}$ and the 


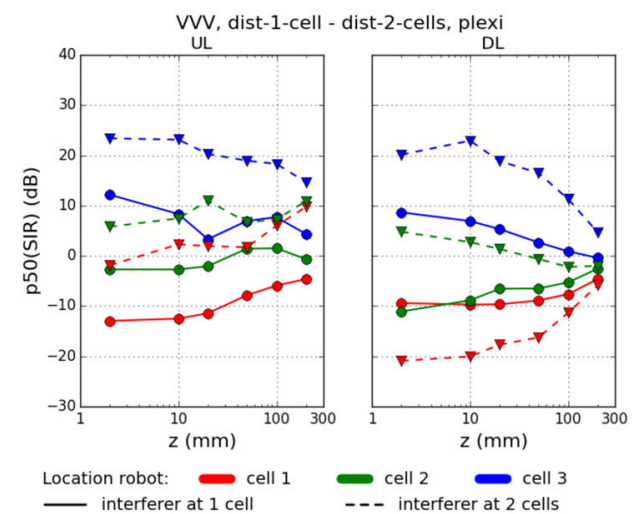

(a)

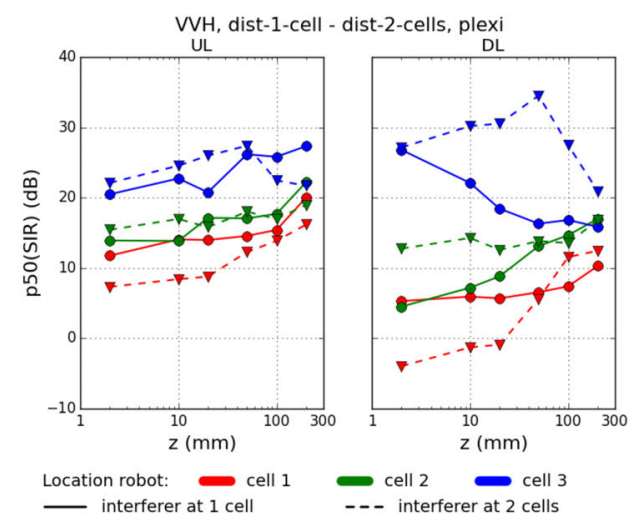

(b)

Figure 7: Influence of the interferer distance on the median of the SIR over the extent of a single cell in a horizontal plane.

\section{Acknowledgments}

PD thanks the ERC for his advanced grant 695495: "ATTO:A new concept for ultra-high capacity wireless networks".

A.T. has received funding from the European Unions Horizon 2020 research and innovation programme under the Marie Skodowska-Curie grant agreement No 665501 with the research Foundation Flanders (FWO). A.T. is an FWO [PEGASUS] Marie Skodowska-Curie Fellow.

\section{References}

[1] B. Lannoo, A. Dixit, D. Colle, J. Bauwelinck, B. Dhoedt, B. Jooris, I. Moerman, M. Pickavet, H. Rogier, P. Simoens, G. Torfs, D. Vande Ginste, and P. Demeester, "Radio-over-Fibre for Ultra-Small 5G Cells ", 17th International Conference on Transparent Optical Networks (ICTON) “, 5-9 July 2015, Budapest, Hungary.

[2] A. Thielens, G. Vermeeren, O. Caytan, G. Torfs, P. Demeester, J. Bauwelinck, H. Rogier, L. Martens, and W. Joseph, "Radiofrequency Exposure near an Attocell as Part of an UltraHigh Density Access Network ", Bioelectromagnetics, 2017 [online available].

[3] O. Caytan, S. Agneessens, S. Lemey, D. Vande Ginste, P. Demeester, and H. Rogier, "Ultra-wideband Cork Substrate-IntegratedWaveguide Cavity-Backed Slot Antenna", International Conference on Electromagnetics in Advanced Applications (ICEAA), 2015 , 7-11 Sept. 2015, Turin, Italy. antenna in each cell was a linearly polarized planar, substrate-integrated-waveguide (SIW) cavitybacked slot antenna. We showed that the interference drops when (1) the interfering cell (with linear polarized antennas) is perpendicularly oriented to the communicating floor cell; (2) the interferer is not in between the moving antenna and the communicating floor cell. Based on the obtained results, we concluded that interference in an atto cell can be significantly reduced by altering the polarization of neighboring floor antennas. Moreover, simultaneous communication in neighboring atto cells is possible and depends on the height of the moving antenna and the mutual orientation of the neighboring cells. Future work will focus on examining the level of the SIR needed to obtain a certain capacity of the network. 